\title{
The Olfactory Strip and Its Preservation in Endoscopic Pituitary Surgery Maintains Smell and Sinonasal Function
}

\author{
Richard J. Harvey ${ }^{1,3}$ Mark Winder ${ }^{1,2}$ Andrew Davidson ${ }^{3} \quad$ Tim Steel $^{2}$ Sunny Nalavenkata ${ }^{1}$ \\ Nadine Mrad ${ }^{1}$ Ali Bokhari ${ }^{1}$ Henry Barham ${ }^{1}$ Anna Knisely ${ }^{1}$ \\ ${ }^{1}$ Department of Rhinology and Skull Base, University of New South \\ Wales, Sydney, Australia \\ ${ }^{2}$ Department of Neurosurgery, St. Vincent's Hospital, Sydney, \\ Australia \\ ${ }^{3}$ Faculty of Medicine and Health Sciences, Macquarie University, \\ Sydney, Australia \\ J Neurol Surg B 2015;76:464-470. \\ Address for correspondence Richard Harvey, MD, PhD, Ground Floor, \\ 67 Burton Street, Darlinghurst NSW 2010, Australia \\ (e-mail: richard@sydneyentclinic.com).
}

\begin{abstract}
Keywords

- smell

- olfaction

- nasoseptal

- endoscopic

- endonasal

- pituitary
\end{abstract}

Differing outcomes on olfaction have been reported from transsphenoidal approaches. ${ }^{1-4}$ In general, patients prefer the endoscopic approach, ${ }^{5}$ and olfactory scores are better after the endoscopic route. ${ }^{6}$ The nasoseptal flap, in particular, to reconstruct the skull base as part of the overall process has been implicated in smell dysfunction. However, much of the literature on the impact of the nasoseptal flap comes from

received

January 10, 2015

accepted after revision

April 11, 2015

published online

June 15, 2015

extended skull base surgery. In our institution, utilizing a small modified nasoseptal flap during simple pituitary surgery has greatly improved our reconstructive options and access. However, controversy exists as to the additional morbidity of utilizing such an approach. The existing studies on patients with large skull base tumors are not an appropriate population to discuss the impact of surgery or

(c) 2015 Georg Thieme Verlag KG Stuttgart · New York
DOI http://dx.doi.org/ 10.1055/s-0035-1554905. ISSN 2193-6331. 
reconstruction because pathology has already dictated much of the morbidity. There is no doubt that resecting large skull base tumors will leave the patient with a new remodeled neosinus cavity that is unlikely to compare with the function of a healthy unoperated unirradiated sinonasal system. Likewise, utilizing the endoscopic endonasal approach to access a giant olfactory groove meningioma or other intracranial tumor is not an appropriate population to assess sinonasal function because the approach results in extensive modification of an otherwise normal anatomy, but it is done to avoid the potential morbidity of frontal lobe retraction. ${ }^{7}$ Additionally, smell loss is anticipated in such patients because the surgical approach or pathology often involves the olfactory apparatus. An ideal study population is the patient undergoing simple transsphenoidal sella-based surgery. Each procedure is relatively comparable, a similar technique is applied each time, and options for exist for the approach via an endonasal endoscopic, transnasal/transseptal/sublabial microscopic method with or without the use of a nasoseptal flap.

The impact of the nasoseptal flap and middle turbinate resection is controversial. ${ }^{3,8,9}$ Unfortunately, standardization of surgical technique does not exist and what is described surgically in some series is not the same as others. In particular, the degree of tissue resection and the location of the nasoseptal flap differ greatly between centers with fellowship-trained rhinologists and those without. Such differences are often noted at scientific meetings during video presentations of techniques.

Rhinologists have been aware of the unique appearance of the upper septal mucosa for some time with the term olfactory strip often used to describe the area (Ricardo Carrau, personal communication), which was noted in editor comments. ${ }^{10}$ Recent study into the impact of the nasoseptal flap on sinonasal quality of life has suggested that modifications need to be made to ensure maximum preservation of sinonasal function; however, there is little doubt that the nasoseptal flap allows for a vascularized graft and enhanced reconstruction compared with free grafting. ${ }^{11}$

This study presents the sinonasal, smell, and objective olfactory outcomes of a standardized olfactory strip preserving nasoseptal flap technique utilized in the endoscopic endonasal transsphenoidal approach to pituitary pathology.

\section{Methods}

A prospective study of olfaction and sinonasal function was undertaken in patients having a nasoseptal flap as part of pituitary surgery. A retrospective cohort with patients undergoing sinonasal tumor surgery was also included. This study was approved by the Hospital Human Research Ethics Committee (SVH09/083). Written informed consent was obtained from all patients.

\section{Population}

Consecutive patients undergoing surgery for pituitary adenomas or simple sella pathology were selected from a tertiary center. Patients with active chronic rhinosinusitis, allergic rhinitis, recreational drug nasal drug use, any regular nasal medication, or a prior history of an olfactory disorder were excluded.

Comparative sinonasal surgery patients were sought. Any patients having a sinonasal tumor removed in which no nasoseptal flap was utilized and where no olfactory apparatus was resected were included. These data were retrospective and part of a previous database on posttumor sinonasal function. ${ }^{12}$

\section{Patient-Reported Outcome Measures}

Four different constructs of patient-reported outcome measures (PROMs) were reported. The Sino-Nasal Outcome Test (SNOT22) was used to assess overall disease-specific quality of life (0-5). This is a validated 22-question survey with four domains: psychological function, sleep function, rhinological symptoms, and ear and/or facial symptoms. ${ }^{13}$ A global rating of sinonasal function on a Likert ordinal scale from -6 (terrible) to 0 (neither good or bad) to +6 (excellent) was also obtained. "Disturbance in smell or taste" was recorded as a 6-point Likert score from 0, "no problem," to 5, "problem as bad as it could be." Nasal symptom scores (NSSs) were recorded via a five-question score from "nasal obstruction," "thick nasal discharge," "facial pain/pressure," "smell disturbance," and "need to blow nose." This was reported as a summary score from 0 to 25 . All four PROMs were recorded at baseline and 6 months postsurgery.

\section{Olfactory Testing}

The Smell Identification Test (SIT40) was utilized. This is a validated scratch-and-sniff olfactory odorant discrimination test reported as dichotomous correct or incorrect smell identification. ${ }^{14,15}$ It was reported as a score from 0 to 40 . The diskettes were scratched and held 2 inches from the nose. There were a closed set of four responses. The test was performed with the patient at rest with no prior food or flavored drink for 30 minutes prior to testing. No prior nasal spray or examination was performed. The test was performed at baseline and at 6 months postsurgery in the pituitary patients only.

\section{Surgical Technique}

A binostril approach with a contralateral port is our standard approach for pituitary work. With this approach the contralateral septal mucosa is nearly completely preserved. No middle turbinate was resected in this approach. After creating the nasoseptal flap (see later), the bone of the septum was removed $2 \mathrm{~cm}$ anterior to the face of the sphenoid or where the posterior septal bone becomes thin. The contralateral mucosa was preserved. The contralateral mucosa was swept laterally from the contralateral face of the sphenoid. The ostium was entered as described earlier but in the submucosal plane. An inferior vertical channel of sphenoid bone was removed on either side of the midline. A large straight Mayo scissor double-action or through-cutting instrument was used to separate the intersinus septum from the roof of the sphenoid. A large grasping forceps was used to remove the sphenoid rostrum. This often came out en bloc, but if not, a drill was used. The remaining face of the sphenoid was 
removed laterally and superiorly to expose the roof and lateral opticocarotid recess (OCR) ( - Fig. 1A). A small opening was made in the elevated mucosa on the contralateral side, incorporating the natural ostium, to allow an instrument to pass through and make binasal surgery possible (-Fig. 1B).

\section{Modified Nasoseptal Flap}

A medium-length needle point monopolar diathermy (Megadyne E-Z Clean 0016AM, Draper, Utah, United States) is used on settings of 12 cut and 12 coagulate power (Force FX-8CS, Valleylab, Boulder, Colorado, United States) to define the flap (-Fig.2). A releasing back incision was made from the choana on the vertical palatine bone (or medial pterygoid) under the sphenopalatine artery (-Fig. 3B). The posterior choana was outlined and the incision continued on the septum 2 to $3 \mathrm{~mm}$ away from its posterior edge to ensure that the incision was down to bone at all times. The incision in the floor of the nose was brought forward at a variable distance laterally on the floor near the inferior turbinate (-Fig. 3C). Then the superior incision started at the superior limit of the sphenoid ostium and the striated thin upper septal mucosa was preserved (-Fig. 3D). The superior septal mucosa is thin, making it less effective for reconstructive purposes, and it contains the olfactory epithelium. The flap was mobilized everywhere but superiorly. The release from the superior edge was made last. The flap was stored in the nasopharynx for later use in reconstruction.

\section{Postoperative Care}

Silastic sheeting $0.51 \mathrm{~mm}$ (Medtronic, Jacksonville, Florida, United States) was used to cover the septum bilaterally. A NasoPore (Polyganics, Groningen, The Netherlands) dressing was utilized within the sphenoid. The patient was allowed to breathe through the nose immediately postoperatively. Mupirocin $2 \%$ ointment and amoxicillin $875 \mathrm{mg} /$ clavulanic acid $125 \mathrm{mg}$ was used twice a day for 10 days. This was intended to reduce staphylococcal co-colonization in the immediate postsurgical period. Large-volume positive-pressure nasal irrigation with commercially prepared buffered isotonic saline was used via a 240-mL squeeze bottle (Sinus Rinse, NeilMed, Santa Rosa, California, United States). This was continued twice daily for 3 weeks at which time the first outpatient review

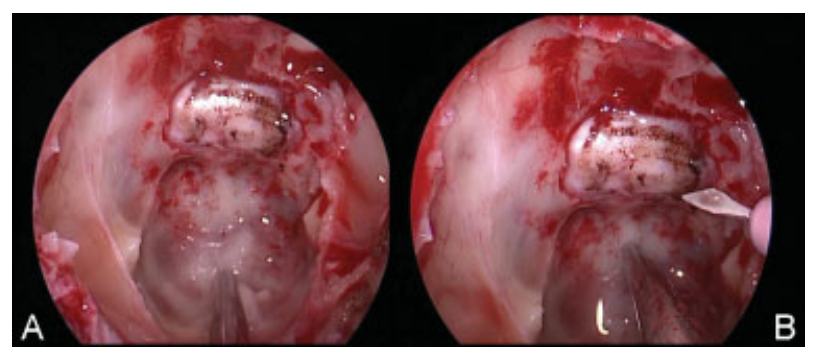

Fig. 1 Our standard exposure is to have one view of all limits of the sphenoid cavity on view. The technique described is not a limited access. The floor is exposed by removal of rostrum and drill to ensure that a straight suction can easily reach the lowest point $(A)$, which allows easy freedom of movement for the surgeon during bimanual dissection (B).

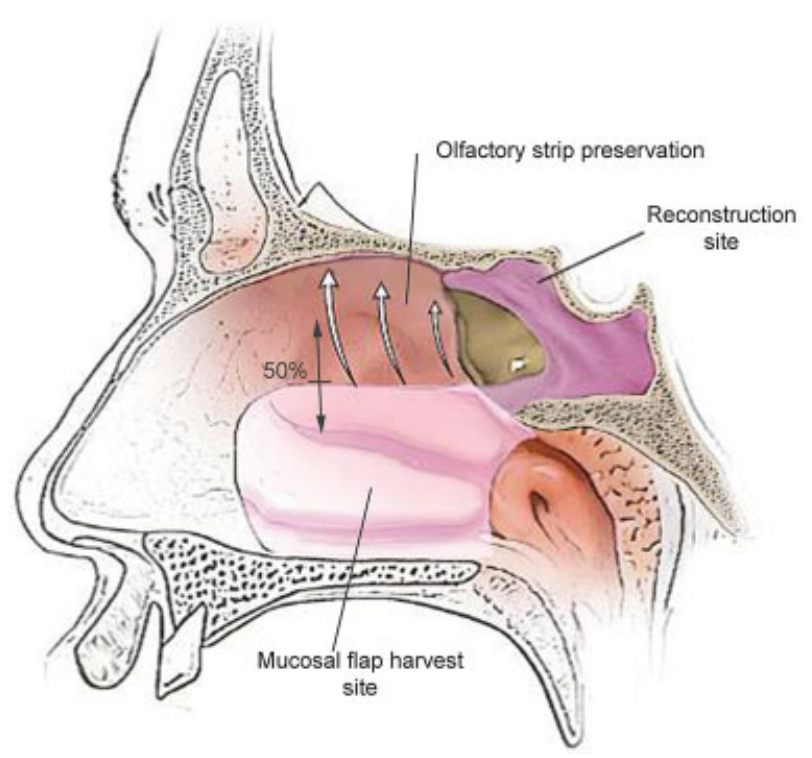

Fig. 2 Design of the olfactory-preserving nasoseptal flap. The olfactory epithelium often has a distinct appearance compared with the mucosal in the lower septum. The mucosal in the lower septum and nasal floor is of better quality for reconstruction.

occurred. The silastic sheets were removed, any residual NasoPore was suctioned, and saline irrigation continued with instructions for daily use decreasing to two to three times weekly, but not to stop, until 90 days postoperatively when most of the healing has occurred. ${ }^{16}$ All sinonasal cavities were examined between 3 and 6 months to check for remucosalization, the absence of crusting, recovery of mucociliary function, and the absence of chronic inflammation (apart from occasional small areas of granulation tissue).

\section{Statistical Analysis}

Statistical analyses were performed using SPSS v.20.0 (Statistical Package for the Social Sciences, Chicago, Illinois, United States).Olfactory, NSSs, and SNOT22 data were considered to be parametric, and the paired Student $t$ test was used to compare preoperative and postoperative scores. Comparisons between response groups were assessed with analysis of variance (ANOVA) and a Bonferroni post hoc analysis for subgroup comparisons. Ordinal data from the smell question and global NSSs were assessed with a Kendal tau-b for changes. All $p$ values were two tailed, and a $p<0.05$ value was considered statistically significant.

\section{Results}

Forty patients (age $50.95 \pm 15.31$ years; $47.5 \%$ female) undergoing pituitary surgery were assessed. - Table 1 presents the baseline nasal function and olfactory data. Approachrelated morbidity was minimal with one patient experiencing a self-resolving epistaxis (2.5\%) and one patient experiencing a cerebrospinal fluid (CSF) leak (2.5\%) requiring exploration and revision of reconstruction. No cases of intracranial bleeding, infection, or new-onset neurologic deficit occurred. All patients were available for their 6-month assessment. 


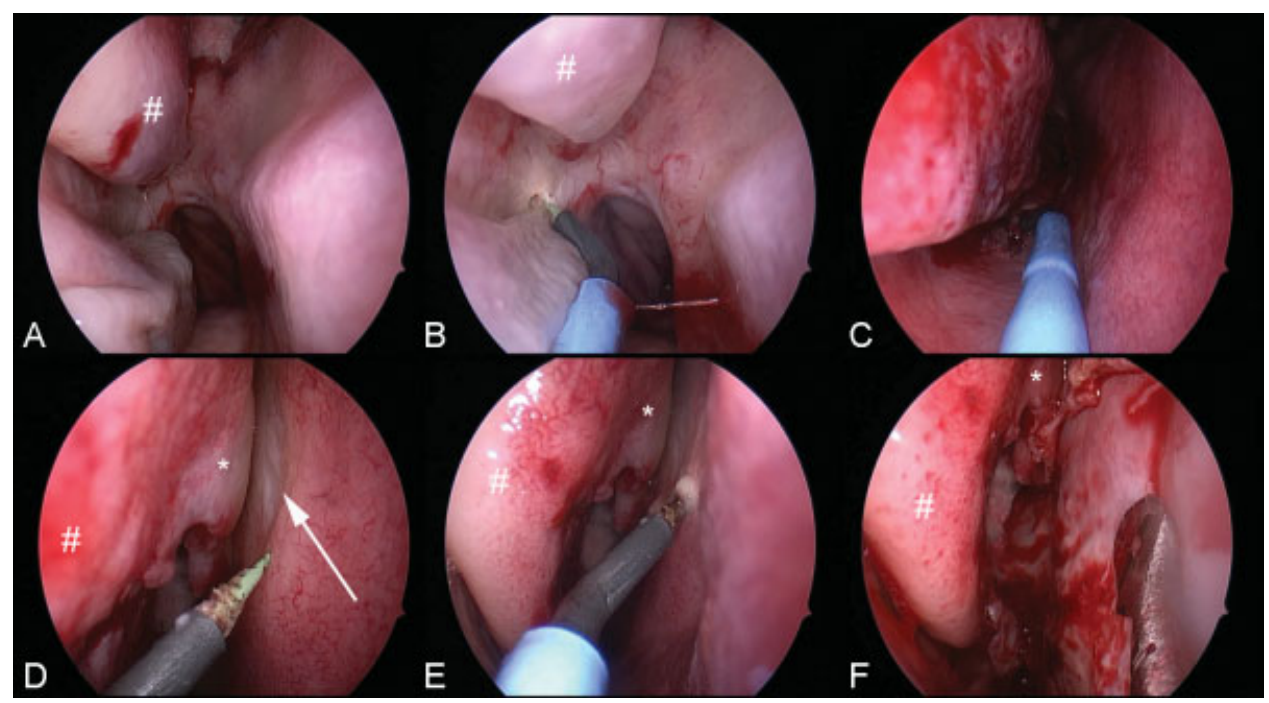

Fig. 3 (A) Approach for a right nasoseptal flap with the middle turbinate (number sign). The incision starts on the medial pterygoid plate (B) and includes the floor (C). The olfactory epithelium (or strip) is often seen as distinct mucosa (arrow) with the superior turbinate (asterisk) and middle turbinate (number sign) close (D). The incision runs below this area (E). The final donor site is only $50 \%$ of the height of the middle turbinate (number sign) (F).

\section{Sinonasal Function and Quality of Life}

For the pituitary patients, the NSSs were not significantly different following pituitary surgery with a nonsignificant lower score at 6 months $(2.75 \pm 3.40$ versus $3.05 \pm 3.03$; $p=0.53)$. Similarly, the SNOT22 scores improved postsurgery $(1.02 \pm 0.80$ versus $0.83 \pm 0.70 ; p=0.046)$. The patients' global assessment of overall nasal function $(-6$ to +6$)$ was statistically better after surgery at 6 months (Kendall tau$\mathrm{b}$ for ordinal scales $p=0.019$ ).

\section{Olfactory Testing in Pituitary Patients}

There was no significant difference in objective smell discrimination scores between baseline and 6 months $(31.63 \pm 3.49$ versus $31.35 \pm 4.61 ; p=0.68)$. The subjective olfactory scores at baseline ( $\boldsymbol{- \text { Table } 1 )}$ ) did change following surgery (Kendall tau-b $p=0.033$ ), but the spread went in both directions, favoring improvement ( - Fig. 4A).

To assess this further, the SIT40 scores of the patients based on a grouping of the change in "Disturbance in smell/ taste" question at 6 months compared with baseline was undertaken. Patients were classified as having a subjective score lower, unchanged, or improved. There was no difference in SIT40 scores between those who subjectively rated their smell lower or higher at 6 months (ANOVA F0.44; $p=0.65$ ) with a post hoc Bonferroni analysis showing no difference on two multiple analyses (-Fig. 4B).

One patient reported a $4+$ decline in function subjectively (-Fig. 5A). This was a 39-year-old acromegalic man who had a suprasellar CSF leak during surgery. He had a clear fluid discharge with a suspected low-pressure headache without meningism at day 2 and was reexplored. There was a clot and Surgicel (Johnson \& Johnson Medical, Norderstedt, Germany) between the planum bone and the nasoseptal flap. The reconstruction was revised with Duragen (Integra LifeSciences, Plainsboro Township, New Jersey, United States) underlay and flap reposition. Surgicel and other material were removed so that the flap made direct contact with the skull base. The sphenoid was packed with iodoform gauze to

Table 1 Baseline data for the study population

\begin{tabular}{|l|l|l|l|}
\hline Outcome & Pituitary $(\boldsymbol{n}=\mathbf{4 0})$ & Sinonasal tumour $(\boldsymbol{n}=\mathbf{5 8})$ & $\boldsymbol{p}$-value \\
\hline Age (yrs) & $50.95 \pm 15.31$ & $52.35 \pm 18.51$ & 0.70 \\
\hline Gender (female, \%) & $47.5 \%$ & $52.5 \%$ & 0.29 \\
\hline SIT 40 & $31.22 \pm 3.72$ & Not performed & n/a \\
\hline Nasal symptom score & $2.75 \pm 3.40$ & $1.46 \pm 0.11$ & 0.01 \\
\hline SNOT22 & $1.02 \pm 0.80$ & $1.27 \pm 0.89$ & 0.16 \\
\hline Global nasal function & 4.0 (IQR 6) & $-2.0($ IQR4) & $<0.01$ \\
\hline Sense of loss of smell or taste & 0 (IQR1) & 0 (IQR3) & 0.02 \\
\hline
\end{tabular}

Note: Global nasal function is scored -6 to +6 , loss of smell or taste is rated 0 to 5 .

Abrreviations: SIT: smell identification test; SNOT22: Sinonasal Outcome Test 22. 

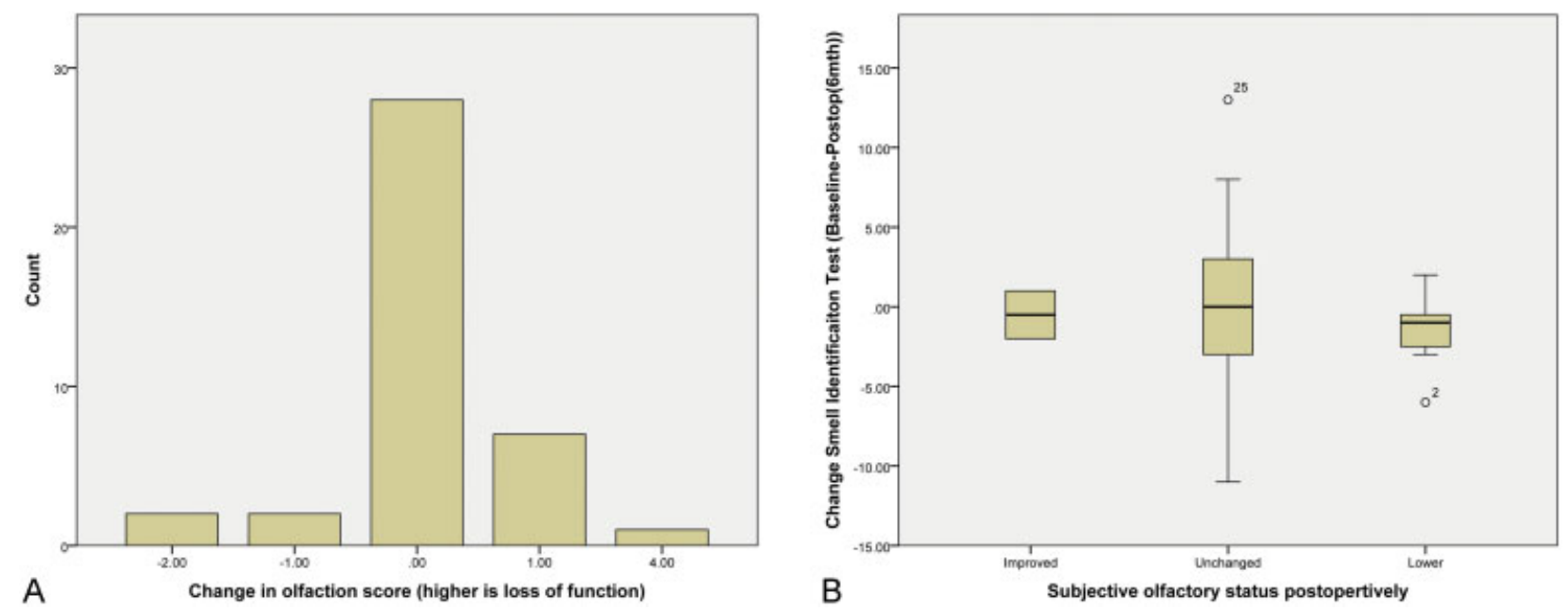

Fig. 4 Frequency for patients and their subjective change in olfaction score (A) and the objective Smell Identification Scores based on the three subjective outcomes (B). There was no difference between groups on Bonferroni two-way post hoc analysis.

ensure that reconstruction layers did not separate again. The recovery was uneventful apart from poor smell at 6 months. On endoscopy, there were only small amounts of granulation at 6 months with a visible olfactory cleft and no adhesions to account for poor smell recovery. Local inflammation was thought to be the cause, and a SIT score of 29/40 (original baseline: $31 / 40$ ) suggested recovery may occur with time.

\section{Subjective Olfaction Compared with Nasal Tumor Surgery Patients}

There were 58 control patients with paranasal tumors (age $52.35 \pm 18.51$ years; $52.5 \%$ female). There was no difference in age $(50.95 \pm 15.31$ years versus $52.35 \pm 18.51$ years; $p=0.70)$ or gender proportions (47.5\% versus $52.5 \%$; $p=0.29$ ) between pituitary and paranasal sinus tumor patients ( - Table $\mathbf{1}$ ). No tumor patient had a septal flap raised. No tumor patient had their olfactory apparatus intentionally resected as part of their procedure. No statistical difference

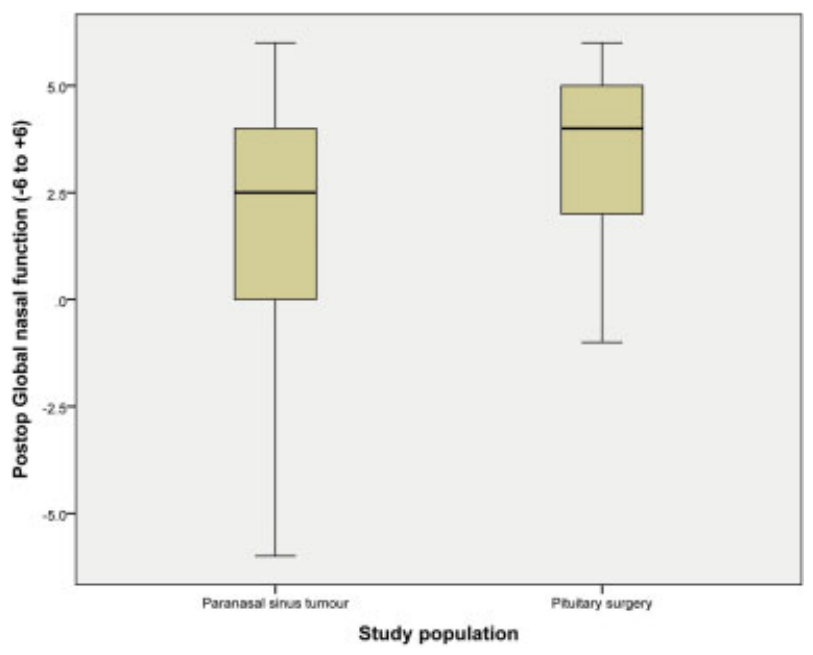

Fig. 5 Overall postoperative rating of global nasal function by patients on a scale of -6 to +6 favors the pituitary group as expected. was seen between the pituitary and paranasal sinus tumor scores for a change in baseline to 6 months olfactory loss (Kendall tau-b $p=0.46$ ).

\section{Discussion}

Rhinologists regularly perform surgery to remove sinonasal tumors, alter paranasal anatomy to access the skull base, and treat inflammatory disease. It is the expectation that when mucosa regenerates and mucociliary function returns, normal sinonasal function will recover. Persistent sinonasal symptoms almost always have a cause, and they do not occur simply because the anatomy was altered. Adhesions can cause mucus trapping if not divided in postoperative care, chronic inflammation can develop, sumps of nonfunction mucosa can be created, and temporary mucus clearance may not be well managed by nasal irrigations. These are common causes of postsurgical sinonasal dysfunction.

Some controversy has arisen regarding the morbidity of nasoseptal flaps, with some groups reporting significant disturbance in smell. ${ }^{3,17}$ The techniques used in these studies may not have preserved the olfactory area as described in this study. Comparison with studies reporting expanded techniques that intentionally transverse the posterior cribriform area do not allow a good comparison of olfactory disturbance because the olfactory morbidity is anticipated as a result of this approach. ${ }^{18}$ Kim et al described differing outcomes based on cold dissection versus electrocautery, ${ }^{19}$ noting that olfactory impairment was uncommon and reported in only one patient with impairment in their series. The premise that thermal injury might contribute highlights the fact that a defined area of olfactory-bearing septal mucosa exists. This was further supported by a repeat study from the Rotenberg group, who originally described significant olfactory disturbance, ${ }^{3}$ and their subsequent study of patients with and without a nasoseptal flap demonstrated that a large flap raised by this group is a detrimental factor. However, the flap described by Tam et al has little respect for the olfactory 
strip on the septum. ${ }^{8}$ In contrast, the technique described in this study utilizes the nasal floor for width. Such mucosa is thick and makes for good reconstruction. Often the entire floor, out to the medial maxillary wall, will be harvested. There is little extra morbidity in this because the greater palatine nerve lies within the descending palatine canal, and the remaining sensation to the posterior palate and teeth arises from the posterior superior alveolar nerve within the infratemporal fossa. No palatal or dental morbidity was seen in our patients, even transiently. This is not true for large flaps in extended skull base surgery that are harvested anteriorly to the squamosa-mucosal junction. The incisive canal can receive either traction or edema, and transient paraesthesia of the incisors can occur.

The olfactory-bearing septal and turbinate area is potentially not as low as many surgeons believe with a prior study demonstrating that only $16 \%$ of the lower third of superior turbinates contain any neuronal elements. ${ }^{20}$ These authors also noted that in the $12 \%$ of patients who reported any subjective disturbance, none of them had neuronal tissue in their specimens to incriminate resection of olfactory mucosa as the cause. Likewise, well-trained rhinologists are able to resect parts of the middle turbinate without affecting olfaction ${ }^{9}$ and note that the impact of surgery on olfaction occurs in the first month but recovers well by 3 months. ${ }^{4}$ Mucosal inflammation does occur from surgical intervention and can be seen on endoscopy. ${ }^{9}$ Some areas that heal by secondary intention can take $\geq 3$ months to fully recover. ${ }^{16}$ This suggests that a combination of minimizing mucosal trauma/inflammation and respecting the olfactory-bearing areas of the nasal cavity is likely to ensure minimal impact on olfaction.

The overall impact of transsphenoidal surgery on sinonasal function was previously assessed. The General Nasal Patient Inventory demonstrated that 3- to 6-month scores returned to baseline for the average patient, but $8 \%$ required ongoing consultation regarding nasal symptoms. ${ }^{2}$ In this study, the baseline nasal symptom scores were higher in the pituitary group despite patients rating their overall sinonasal function as better in the pituitary group. This is difficult to explain, but the $30 \%$ rate of acromegaly in our group (12 of 40 ) might account for nasal symptoms at baseline. As expected, the final sinonasal function favored the pituitary group (-Fig. 5).

Tissue manipulation and trauma can be minimal with an endoscopic approach compared with microscopic access, which is reflected in patient preference ${ }^{5}$ and in recovery of function. ${ }^{1,6}$ Ultimately, this mucosal trauma is dictated by differences in surgical technique, even among procedures described under the umbrella term endonasal endoscopic approach" However, the design of the nasoseptal flap has not been well addressed, and it is likely that heterogeneous practices exist. Authors have suggested modifications to the technique, ${ }^{1,17}$ and the data presented in this study provide evidence that preservation of the olfactory strip as a discrete area can avoid significant impact on both olfaction and sinonasal function. Although the concept of an olfactory strip was promoted by colleagues (e.g., Ric Carrau) for many years and alluded to in publications, ${ }^{21}$ the focus was usually on septal mucosal recovery. Since then, the observation that mucosal regeneration occurs much more quickly under silastic sheeting ${ }^{22}$ with or without free mucosa grafts ${ }^{23}$ has shifted the focus away from debate over donor site morbidity. Only one comment to the editor could be found in the literature that discussed this unique area in relation to pituitary surgery. $^{10}$

A modified nasoseptal flap with preservation of the olfactory strip can provide a low morbidity approach while maintaining reconstruction options. Such a flap can provide better reconstruction than free grafts, ${ }^{11}$ and it can easily be reused as part of future surgical interventions. ${ }^{24}$ Such options are potentially more important in the management of pituitary adenomas if complete resection is not the intended goal from initial surgery and further surgical interventions are considered likely in the future.

\section{Conclusion}

Separating the effects of postsurgical inflammation from the impact of approach is always difficult. Surgeons have differing approaches to mucosal preservation, surgical technique, and postoperative care. However, preserving the olfactory strip of septal mucosa can provide a low morbidity approach while maintaining reconstruction options.

Conflicts of Interest

Richard J. Harvey has served on an advisory board for Schering Plough and Glaxo-Smith-Kline; served as a previous consultant with Medtronic, Olympus, and Stallergenes; participated in the speakers' bureau for Merck Sharp \& Dohme and ArthroCare; and he has received grant support from NeilMed. The remaining authors have nothing to disclose.

Funding

This study was investigator initiated and received no industry funding.

\section{References}

1 Kim B-Y, Son HL, Kang S-G, et al. Postoperative nasal symptoms associated with an endoscopic endonasal transsphenoidal approach. Eur Arch Otorhinolaryngol 2013;270(4):1355-1359

2 Wang YY, Srirathan V, Tirr E, Kearney T, Gnanalingham KK. Nasal symptoms following endoscopic transsphenoidal pituitary surgery: assessment using the General Nasal Patient Inventory. Neurosurg Focus 2011;30(4):E12

3 Rotenberg BW, Saunders S, Duggal N. Olfactory outcomes after endoscopic transsphenoidal pituitary surgery. Laryngoscope 2011;121(8):1611-1613

4 Hart CK, Theodosopoulos PV, Zimmer LA. Olfactory changes after endoscopic pituitary tumor resection. Otolaryngol He ad Neck Surg 2010;142(1):95-97

5 Lwu S, Edem I, Banton B, et al. Quality of life after transsphenoidal pituitary surgery: a qualitative study. Acta Neurochir (Wien) 2012; 154(10):1917-1922 
6 Kahilogullari G, Beton S, Al-Beyati ESM, et al. Olfactory functions after transsphenoidal pituitary surgery: endoscopic versus microscopic approach. Laryngoscope 2013;123(9):2112-2119

7 de Almeida JR, Carvalho F, Vaz Guimaraes Filho F, et al. A comparison of postoperative MRI signal changes between endoscopic endonasal approaches and open approaches for olfactory groove meningiomas: a match paired analysis from two institutions. J Neurol Surg B (Suppl BS1):2014:S19

8 Tam S, Duggal N, Rotenberg BW. Olfactory outcomes following endoscopic pituitary surgery with or without septal flap reconstruction: a randomized controlled trial. Int Forum Allergy Rhinol 2013;3(1):62-65

9 Sowerby LJ, Gross M, Broad R, Wright ED. Olfactory and sinonasal outcomes in endoscopic transsphenoidal skull-base surgery. Int Forum Allergy Rhinol 2013;3(3):217-220

10 Simal Julián JA, Miranda Lloret P, Pancucci G, Sanromán Álvarez P, Botella Asunción C. Avoiding olfactory impairment after endoscopic endonasal expanded approaches. Neurosurgery 2013; 73(3):E562-E563

11 Harvey RJ, Parmar P, Sacks R, Zanation AM. Endoscopic skull base reconstruction of large dural defects: a systematic review of published evidence. Laryngoscope 2012;122(2):452-459

12 Harvey RJ, Malek J, Winder M, et al. Sinonasal morbidity following tumour resection with and without nasoseptal flap reconstruction. Rhinology 2014. In press

13 Hopkins C, Gillett S, Slack R, Lund VJ, Browne JP. Psychometric validity of the 22-item Sinonasal Outcome Test. Clin Otolaryngol 2009;34(5):447-454

14 Doty RL, Genow A, Hummel T. Scratch density differentiates microsmic from normosmic and anosmic subjects on the University of Pennsylvania Smell Identification Test. Percept Mot Skills 1998;86(1):211-216

15 Doty RL, Shaman P, Dann M. Development of the University of Pennsylvania Smell Identification Test: a standardized microen- capsulated test of olfactory function. Physiol Behav 1984;32(3): 489-502

16 Jo HW, Dalgorf DM, Snidvongs K, Sacks R, Harvey RJ. Postoperative irrigation therapy after sinonasal tumor surgery. Am J Rhinol Allergy 2014;28(2):169-171

17 Alobid I, Enseñat J, Mariño-Sánchez F, et al. Expanded endonasal approach using vascularized septal flap reconstruction for skull base tumors has a negative impact on sinonasal symptoms and quality of life. Am J Rhinol Allergy 2013;27(5):426-431

18 Alobid I, Enseñat J, Mariño-Sánchez F, et al. Impairment of olfaction and mucociliary clearance after expanded endonasal approach using vascularized septal flap reconstruction for skull base tumors. Neurosurgery 2013;72(4):540-546

19 Kim S-W, Park KB, Khalmuratova R, Lee H-K, Jeon S-Y, Kim DW. Clinical and histologic studies of olfactory outcomes after nasoseptal flap harvesting. Laryngoscope 2013;123(7):1602-1606

20 Say P, Leopold D, Cochran G, Smith L, Greiner T. Resection of the inferior superior turbinate: does it affect olfactory ability or contain olfactory neuronal tissue? Am J Rhinol 2004;18(3): 157-160

21 Rivera-Serrano CM, Snyderman CH, Gardner P, et al. Nasoseptal "rescue" flap: a novel modification of the nasoseptal flap technique for pituitary surgery. Laryngoscope 2011;121(5):990-993

22 Phillips PS, Harvey RJ. Large skull base defect reconstruction with and without pedicled flaps. In: Palmer JN, Chiu A, eds. Atlas of Endoscopic Sinus and Skull Base Surgery. Philadelphia, PA: Elsevier; 2012

23 Kimple AJ, Leight WD, Wheless SA, Zanation AM. Reducing nasal morbidity after skull base reconstruction with the nasoseptal flap: free middle turbinate mucosal grafts. Laryngoscope 2012;122(9): 1920-1924

24 Zanation AM, Carrau RL, Snyderman CH, et al. Nasoseptal flap takedown and reuse in revision endoscopic skull base reconstruction. Laryngoscope 2011;121(1):42-46 